\title{
Study on Beamforming V2I Scenarios for Sub-6 GHz and mmWave Channels
}

\author{
Christian Ballesteros*, Germán Ramírez*†, Luca Montero*, Jordi Romeu*, Luis Jofre* \\ * Signal Theory and Communications Dept, Universitat Politècnica de Catalunya (UPC), Barcelona, Spain, \\ \{christian.ballesteros, romeu, jofre\}@tsc.upc.edu, luca.montero@upc.edu \\ † Universidad Nacional de Colombia, Bogotá, Colombia, garamirezar@unal.edu.co
}

\begin{abstract}
The study of the wireless channel between a hybrid massive MIMO Base Station (BS) and a vehicular platform is proposed. Several multi-antenna geometries and MIMO architectures in both vehicle and BS are numerically modeled and compared. Different metrics are used for the assessment of the system performance, including channel capacity, in two frequency bands, sub-6 GHz $(5.9 \mathrm{GHz})$ and millimeter-wave (mmWave) $(26 \mathrm{GHz})$, under different propagation conditions. The use of beamforming techniques on the vehicle side is compared to conventional SISO and MIMO solutions. In the urban scenario used in the study, a $45^{\circ}$ beamwidth circular array is able to enhance the single monopole performance up to $157 \%$ in capacity, and outperform MIMO $4 \times 4$ in most situations.
\end{abstract}

Index Terms-Massive MIMO, beamforming, propagation, vehicle.

\section{INTRODUCTION}

Next-generation networks need to account for the introduction of such a new and challenging agent which are connected vehicles, including a wide variety of services and technologies such as traffic safety, roadside assistance, infontainment or autonomous vehicles. All those scenarios are included in Vehicle-to-Everything (V2X) communications. In particular, Vehicle-to-Infrastructure (V2I) [1] enable real-time sensor data sharing, multimedia streaming and 3D mapping with high demands in terms of latency and bandwidth.

Those requirements are expected to be barely covered by conventional technologies based on sub- $6 \mathrm{GHz}$ carriers, such as 5.9 GHz IEEE 802.11p Dedicated Short Range Communications (DSRC) [2]. In this case, mmWave bands appear to play a key role in future networks. European $5 \mathrm{G}$ New Radio (NR) between 24.25 and $27.5 \mathrm{GHz}$ is the first milestone but upper bands are also into consideration, for example, in the $57-66 \mathrm{GHz}$ or $77-81 \mathrm{GHz}$ range [3]-[5].

The coexistence of $5.9 \mathrm{GHz}$ DSRC with mmWave-V2X communications can take advantage for example of IEEE 802.11 , which defines standard messages with information directly taken from on-board sensors including vehicle position, speed, acceleration, and path prediction, that could be used to enhance and alleviate the processing burden in new V2X applications. Also the congruence in temporal and angular domains between sub- $6 \mathrm{GHz}$ and mmWave bands can be beneficially exploited [6].

In contrast to the previous generation, massive MIMO (mMIMO) for mmWave [7] proposes two alternatives to increase channel capacity based on the exploitation of the multiple signal paths and the computational power available at the modern BS. For that purpose, it is envisioned that, as a first alternative, the BS antenna performs beamforming focusing energy only on its intended set of receivers. One step beyond, in combination with proper channel estimation, the BS should be able to send delayed copies of information through the redundant multiple paths available in a way that reach their destination coherently.

Assessing the performance of these systems in realistic deployment scenarios [8] involves long duration and high cost site-specific measurement campaigns. This approach is not practical in most cases, therefore computational techniques are a suitable alternative [9].

This manuscript presents a study of an urban V2I channel for two frequency bands: 5.9 and $26 \mathrm{GHz}$. A methodology based on channel parameters is defined to properly compare both frequencies, including received power, channel eigenvalues and the achievable ergodic capacity. The analysis is performed for three different cases according to the antenna configuration on the BS and the car: a massive single-beam $\mathrm{BS}$, namely massive SISO (mSISO), with a monopole on top of the vehicle; the same massive BS with a circular array of 16 elements on the vehicle for single-beam beamforming, $\mathrm{mSISO}$ Beamforming (mSISO-BF), and a modular subdivision of the massive BS into four arrays and four monopoles on the car, i.e., $\mathrm{mMIMO} 4 \times 4$.

\section{Channel Model}

This work relates a downlink communication between a massive BS and a vehicle. The number of RF streams per side will define the MIMO order and the channel matrix, $\mathbf{H}$, dimensions. In general, it consists of $\mathrm{N}_{\mathrm{T}} \times \mathrm{N}_{\mathrm{R}}$ entries, being $\mathrm{N}_{\mathrm{T}}$ the number of modules at the $\mathrm{BS}$ and $\mathrm{N}_{\mathrm{R}}$ the RF ports on the vehicle.

The propagation simulation is based on the ray-tracing approach in which transmitter and receiver antenna far-field patterns are used to set the angular weight of the outgoing and incoming rays. Each ray suffers different reflection and diffraction processes, but diffuse scattering is not considered. In this way, the transmitter is properly modeled as long as any object intercepting a launched ray is located beyond the far field region of the antenna. The receiving antennas are modeled in presence of the vehicle platform. 


\section{A. Performance Metrics}

We know from [10] that the ergodic capacity can be written as:

$$
C=\log _{2}\left(\operatorname{det}\left[\boldsymbol{I}+\frac{P_{T} \boldsymbol{H} \boldsymbol{H}^{*}}{\sigma^{2}}\right]\right),
$$

where $\mathbf{P}_{\mathrm{T}}$ and $\sigma^{2}$ are transmit and noise power, respectively, $\mathbf{I}$ is the identity matrix, and $(.)^{*}$ denotes the transpose conjugate. Each entry in the channel matrix includes the transfer function for all combinations of MIMO ports, which correspond to the addition of the different multipath contributions from the elements present in the scenario.

A measure of the channel diversity and scattering properties of the environment is based on its eigenvalues. They define the maximum number of usable data streams to be sent through the channel. Hence, the higher the number of relevant eigenvalues, the larger the MIMO order to make use of those data streams. In that terms, the channel capacity can be also expressed as the sum of the individual capacities of those streams weighted by their associated eigenvalues $\lambda_{i}$.

$$
C=\sum_{i=1}^{N} \log _{2}\left(1+\lambda_{i} S N R\right)
$$

In (2), the Signal to Noise Ratio (SNR) is referred at the end of the receiving chain $\left(\mathrm{SNR}=\mathrm{P}_{\mathrm{R}} / \sigma^{2}\right)$.

For all cases of study, the BS will be pointing towards some reference positions in which the vehicle is expected to be located. When the beamforming is used on the vehicle, it focuses in the same direction towards the BS. For each reference position, a focusing area proportional to the $-3 \mathrm{~dB}$ beamwidth of the BS is defined around the particular point. The average capacity inside this region is calculated from a total number of $\mathrm{N}_{\mathrm{S}}$ samples.

$$
C_{a v .}=\frac{1}{N_{S}} \sum_{i=1}^{N_{S}} C_{i}
$$

\section{B. Hybrid Masssive MIMO Base Station}

A planar massive BS is proposed. A total of $\mathrm{Q}$ antennas is divided in $\mathrm{M}$ rows and $\mathrm{N}$ columns. In SISO operation, all $\mathrm{Q}$ elements are weighted to create a single beam towards the user, whereas MIMO $4 \times 4$ operation consists on a columnwise BS division into four modules of $\mathrm{M} \times \mathrm{P}$ antennas, with an $\mathrm{RF}$ input each. Fig. 1 depicts the geometry previously described.

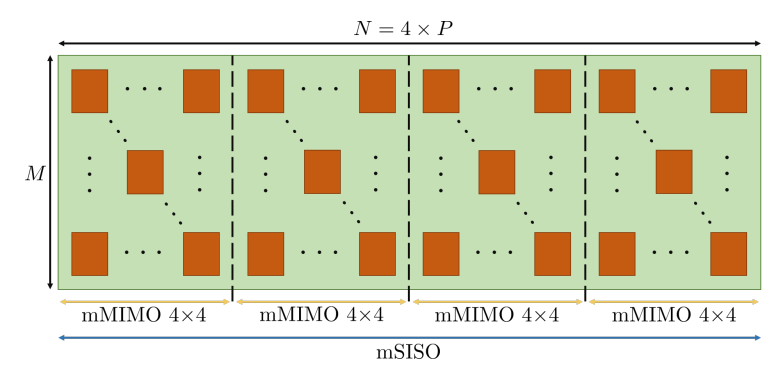

Fig. 1. Massive BS with $\mathrm{M} \times \mathrm{N}$ patch antennas and the modular divisions for the $\mathrm{mMIMO}$ configuration.
Two different frequency bands are compared in terms of their electromagnetic properties. Given the propagation loss increase with frequency, larger gain is required to partially compensate this effect. In this regard, two BS geometries are considered for the two frequencies of interest, trying to maintain similar values of the total physical area. At $5.9 \mathrm{GHz}$, the geometry is composed of $4 \times 16$ elements $(\mathrm{Q}=64)$, whereas, at $26 \mathrm{GHz}$, the antennas are scaled to fit $16 \times 128$ patches $(\mathrm{Q}=2048$ elements in total). For both frequencies, the available power is fixed to $0 \mathrm{dBm}$.

The radiated beams are generated with uniform amplitude and a proper phase according to the focusing direction. Phased arrays are the most extended beamforming architectures given their lowest power consumption compared to other more complex strategies. Perfect knowledge of the vehicle position is assumed in the calculation. Fig. 2 shows the azimuth and elevation gain patterns for both frequencies when using the mSISO arrangement pointing towards a reference point $\left(\theta=101^{\circ}\right.$ and $\left.\phi=71^{\circ}\right)$.

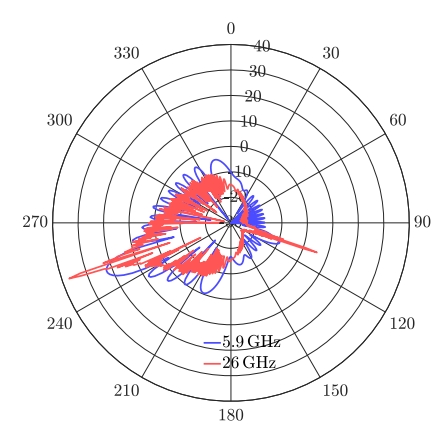

(a) Azimuth pattern for $\theta=101^{\circ}$.

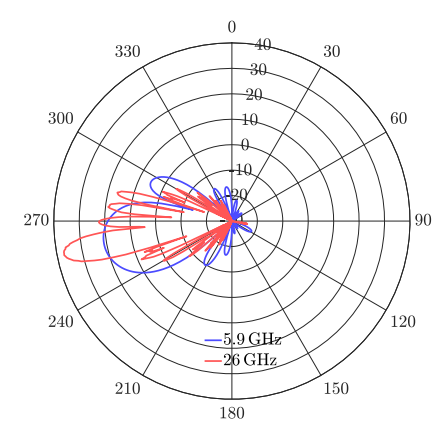

(b) Elevation pattern for $\phi=71^{\circ}$.
Fig. 2. Gain polar patterns of the massive BS when pointing towards one of the reference points in free space at 5.9 and $26 \mathrm{GHz}$.

\section{Multi-antenna Vehicle}

The receiver is a vehicle describing a certain trajectory in the urban scenario later described. Three options are considered regarding the antenna configuration

- A single quarter-wavelength monopole mounted on the center of the car roof (mSISO, beamforming capabilities only at the massive BS).

- An array of 16 monopoles arranged along a circumference with a diameter of one wavelength. It is intended to attain 8 independent patterns, every $45^{\circ}$, in the horizontal plane, with an elevation angle from zenith of $\theta=80^{\circ}$, used for the mSISO-BF case.

- 4 monopoles, assumed uncoupled, disposed on a line in the longitudinal axis of the vehicle, for mMIMO $4 \times 4$.

Fig. 3 represents the different antenna configurations on the vehicle side. The maximum gain at $5.9 \mathrm{GHz}$ of the monopole centered on the roof is $7.7 \mathrm{~dB}$, whereas for the circular array is $13.4 \mathrm{~dB}$. Similar values are achieved at $26 \mathrm{GHz}$, since the antenna geometry is properly scaled to the wavelength. 


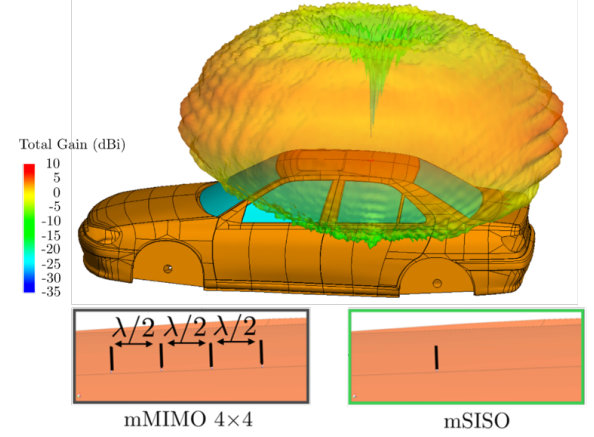

(a) Single monopole pattern.

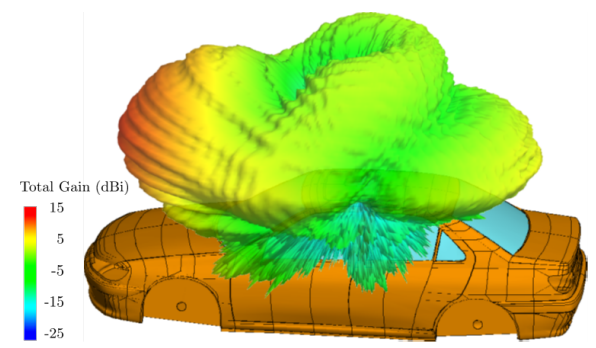

(b) 16-element array pointing to the front direction.

Fig. 3. 3D gain patterns at $5.9 \mathrm{GHz}$ of a single monopole (used in $\mathrm{mSISO}$, similar to displaced mMIMO $4 \times 4$ monopoles) and the 16-element array.

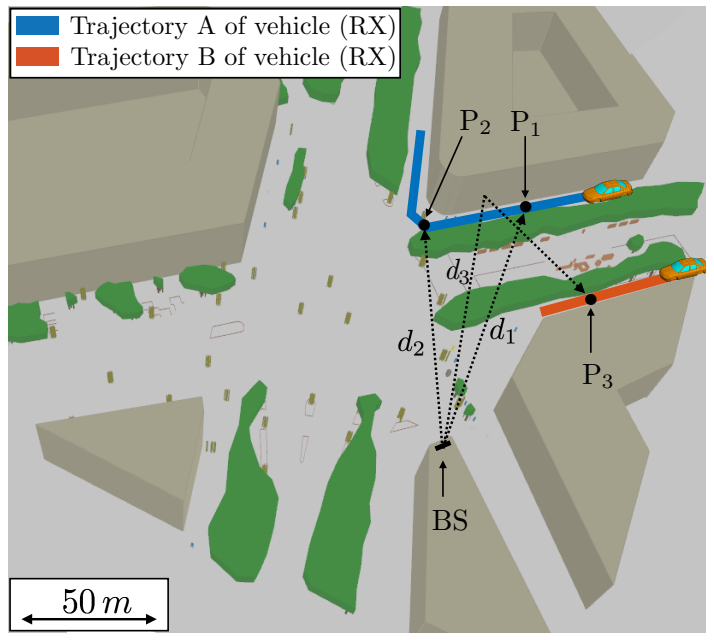

Fig. 4. The scenario of the intersection in Barcelona.

\section{Urban V2I Scenario}

A realistic crossroad in the city of Barcelona is recreated, as shown in Fig. 4. The environment is composed of an asphalt plane upon which five concrete buildings with a height of $18 \mathrm{~m}$ are located. In addition, properly modeled common obstructing objects are scattered around, e.g., metallic traffic lights and trees composed of a wooden trunk plus a vegetation layer on top.

The BS is placed on top of a building at $21 \mathrm{~m}$ from the ground. The car, $1.42 \mathrm{~m}$ height, describes two trajectories (namely A and B) sampled every $0.2 \mathrm{~m}$ under different visibility conditions with respect to the BS. Trajectory A is $140 \mathrm{~m}$ long and the Line of Sight (LOS) path is not blocked by any building, but the first meters are obstructed by the vegetation that partially attenuates the signal, i.e., Obstructed Line of Sight (OLOS). Trajectory B is $55 \mathrm{~m}$ long and completely in Non-Line of Sight (NLOS).

Three reference points $\left(\mathrm{P}_{1}, \mathrm{P}_{2}, \mathrm{P}_{3}\right)$ are defined under different visibility conditions to validate the focusing capabilities of both the BS and the car (in mSISO-BF). For points $\mathrm{P}_{1}$ and $\mathrm{P}_{2}$, in trajectory $\mathrm{A}$, the focusing strategy is concentrated in the direct path between the transmitter and the receiver. For point $\mathrm{P}_{3}$, in trajectory $\mathrm{B}$, the beam is pointed towards the opposite wall, corresponding to the strongest reflection.

\section{RESULTS}

The performance of the three antenna configurations under study (mSISO, mSISO-BF and mMIMO $4 \times 4$ ) is studied in terms of power, channel eigenvalues and average capacity in the focusing area. This region is defined as an interval within the trajectory centered on the three reference points according to the scenario conditions and the $-3 \mathrm{~dB}$ beamwidth for the mSISO case at both frequencies in the most restrictive dimension. At $5.9 \mathrm{GHz}$, it is translated to an interval of $30 \mathrm{~m}$, whereas at $26 \mathrm{GHz}$ the interval is reduced to $8 \mathrm{~m}$. The noise level is assumed constant at $-90 \mathrm{dBm}$.

\section{A. Received power}

The power at the receiver (vehicle) is depicted in Fig. 5 for the two frequencies into consideration, 5.9 and $26 \mathrm{GHz}$, for the single beam case. The BS always focuses the power towards the reference positions along the two trajectories, but two cases are assumed on the car. First, using the single monopole centered on the roof, no beamforming is used. Then, focusing capabilities are introduced in the vehicle by means of a 16-element circular array with $45^{\circ}$ resolution. The values have been averaged using a sliding window of five samples to reduce the fast fading effect in the plot.

For the two points in trajectory A, Fig. 5a and 5b, the fields are really concentrated around the reference points, especially for the highest frequency with higher resolution given the larger dimensions of the BS in terms of wavelength. The higher directivity also compensates the additional free space losses, reaching similar power values within the focusing areas at both frequencies. In addition, the use of the circular array increases the received power between 5 and $10 \mathrm{~dB}$.

\section{B. MIMO Channel Eigenvalues}

In a Multiple Input Multiple Output (MIMO) system, the channel diversity determines the final improvement in terms of capacity with respect to the use of a single radio channel. The eigenvalues are analyzed for the two trajectories when the BS is horizontally divided in four modules at the two frequencies, 5.9 and $26 \mathrm{GHz}$. The beamwidth is increased with the division because less elements are used to create each beam, despite precoding techniques could alleviate this effect.

Fig. 6 represents the Frobenius-normalized eigenvalues at $5.9 \mathrm{GHz}$ for both trajectories. The values have also been averaged using a five-sample sliding window for representation. 


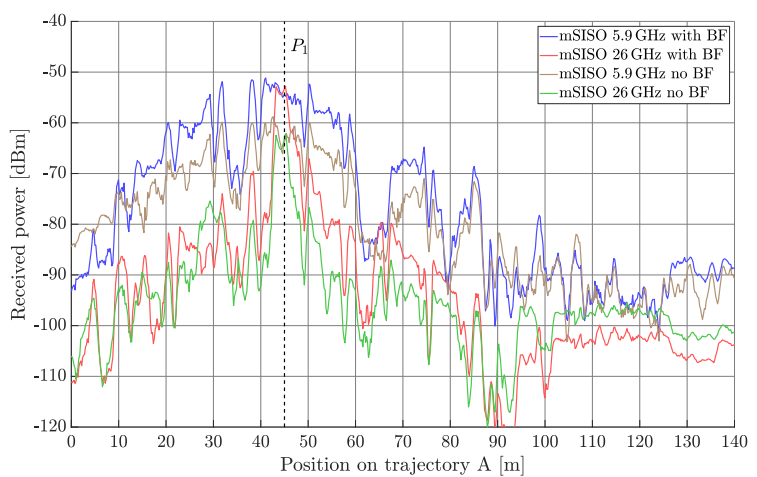

(a) BS pointing to $P_{1}$.

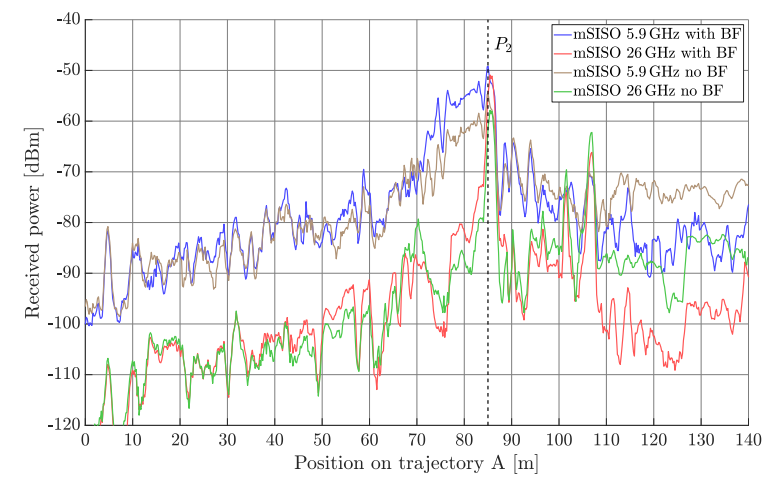

(b) BS pointing to $\mathrm{P}_{2}$.

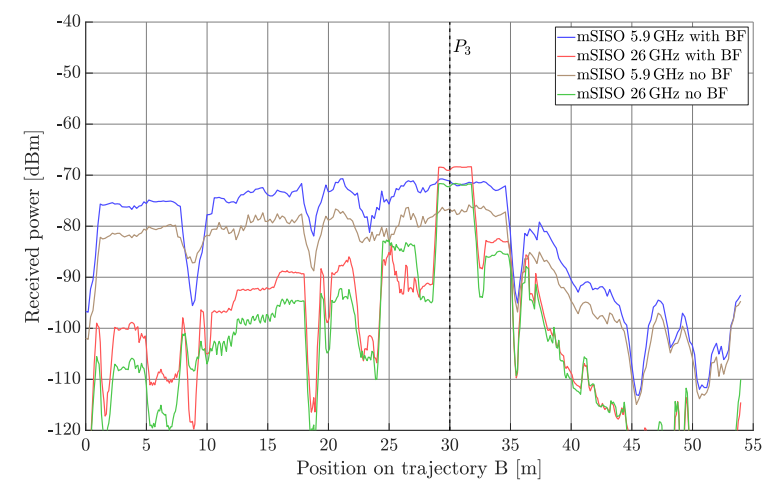

(c) BS pointing to $\mathrm{P}_{3}$.

Fig. 5. Received power for the two frequencies, 5.9 and $26 \mathrm{GHz}$, with and without beamforming on the vehicle and single beam BS.

The highest value is always below 0.9 and there is also a second significant value. For relatively high SNR values, one can benefit from MIMO to increase capacity, even in the case of LOS visibility.

Fig 7 represents the windowed eigenvalues for the highest frequency, $26 \mathrm{GHz}$. The strongest value is clearly above 0.8 or even 0.9 , except outside the focusing regions. The largest gain of the BS and the directional nature of the mmWave region [11] lead to a dominant eigenvalue.

\section{Average Capacity}

The final performance of the system is validated by the average capacity in the focusing area for each reference point.

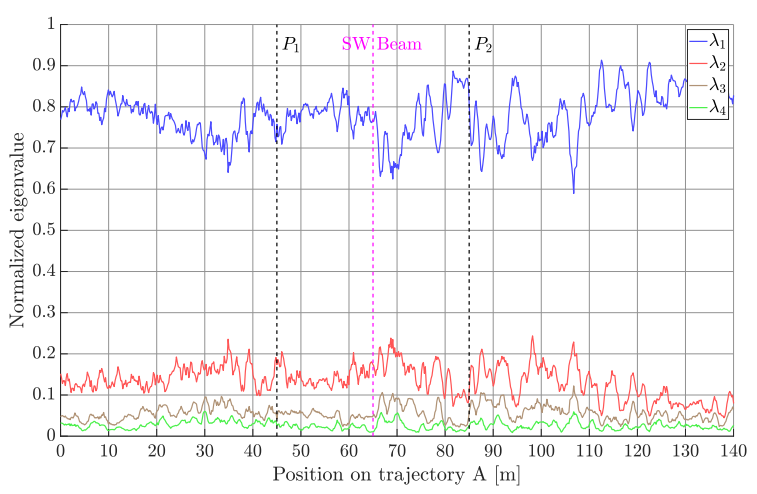

(a) Trajectory A.

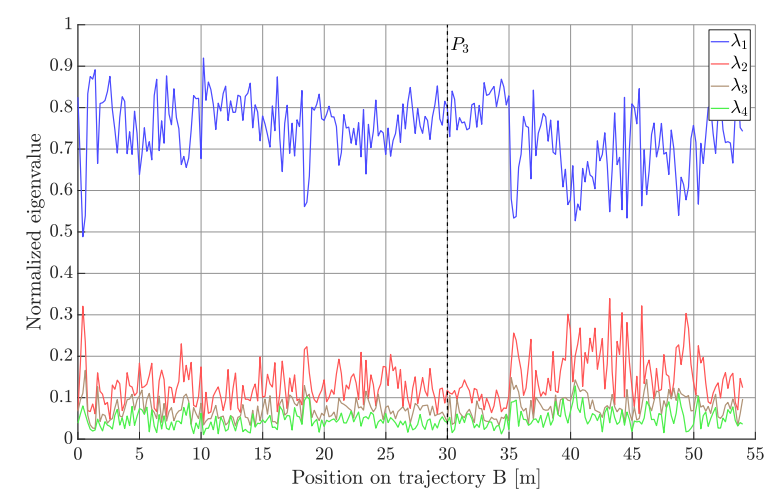

(b) Trajectory $\mathrm{B}$.

Fig. 6. Normalized eigenvalues at $5.9 \mathrm{GHz}$ for both trajectories. In trajectory $\mathrm{A}$, the beam is switched between both reference points.

Fig. 8 represents the values for the two frequencies and the three points using the different antenna configurations: mSISO using the single monopole on the car, mSISO with car beamforming and mMIMO $4 \times 4$.

At the lowest frequency of $5.9 \mathrm{GHz}$, Fig. 8a, one can state two main facts: using beamforming on the car improves capacity under any visibility condition and it can even outperform mMIMO, especially in NLOS. In average, as seen in Fig 5, the received power is larger in the focusing area of $\mathrm{P}_{1}$, which leads to a high SNR regime. This fact explains the largest capacity for similar eigenvalue distributions.

The previous explanation cannot be extended to $26 \mathrm{GHz}$, as seen in Fig. 8b. The narrower beam of the BS and the additional losses in the multipath components at higher frequencies result in a more unequal distribution of the eigenvalues in the focusing area and lower average power. The unitary bandwidth capacity (bps/Hz) is always lower compared to $5.9 \mathrm{GHz}$, but the use of beamforming techniques on the car is able to improve capacity in all cases with respect to mMIMO $4 \times 4$.

When comparing both frequencies, the additional losses in the mmWave band result in lower capacity with respect to the sub-6 GHz technologies. Nevertheless, it must be mentioned that they are normalized to the bandwidth and the final achievable throughput will be higher in the mmWave band. The expected channel bandwidth in 5G NR FR2 varies from 


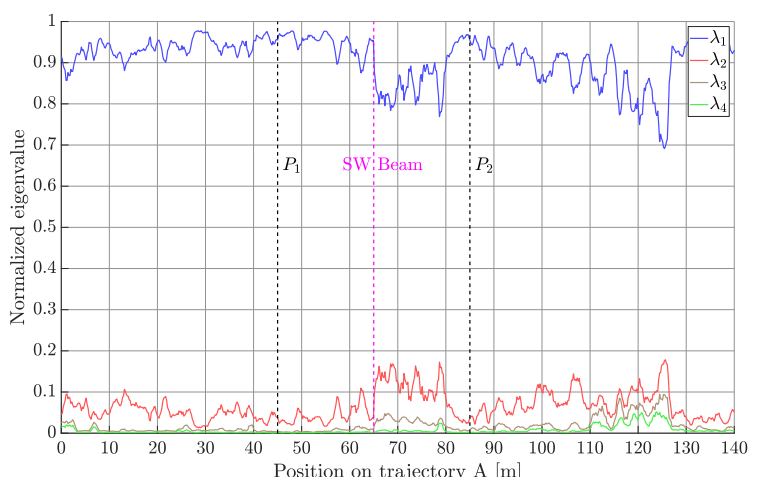

(a) Trajectory A.

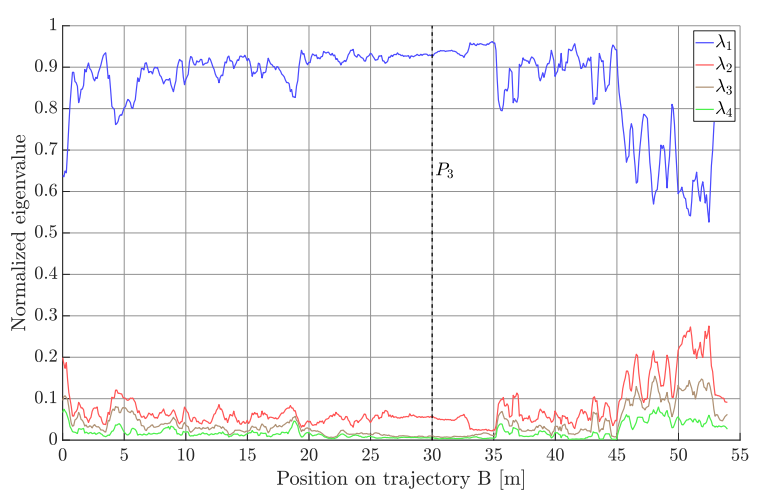

(b) Trajectory B.

Fig. 7. Normalized eigenvalues at $26 \mathrm{GHz}$ for both trajectories. In trajectory $\mathrm{A}$, the beam is switched between both reference points.

50 to $400 \mathrm{MHz}$, whereas $802.11 \mathrm{p}$ typically uses $10 \mathrm{MHz}$. This implies an increment of at least five times of bandwidth.

\section{CONCLUSION}

The performance of different mMIMO modalities has been analyzed. The extra propagation losses at higher frequencies require massive geometries and accurate beamforming techniques. The use of beamforming on the vehicle has been demonstrated to outperform both the single and the multiple antenna performance in their conventional manner (without focusing). This statement is especially true at high frequencies, whose directive nature lead to more attenuated multipath components make single beam focusing techniques more suitable. In sub- $6 \mathrm{GHz}$ bands, a modular decomposition of the massive BS architecture is able to improve substantially the channel capacity in high SNR situations.

\section{ACKNOWLEDGMENT}

This work was supported by the Spanish Comision Interministerial de Ciencia y Tecnología (CICYT) under projects TEC2016-78028-C3-1-P, MDM2016-0600, and Catalan Research Group 2017 SGR 219. The Spanish Ministry of Education contributes via a doctoral grant to the first author (FPU17/05561).

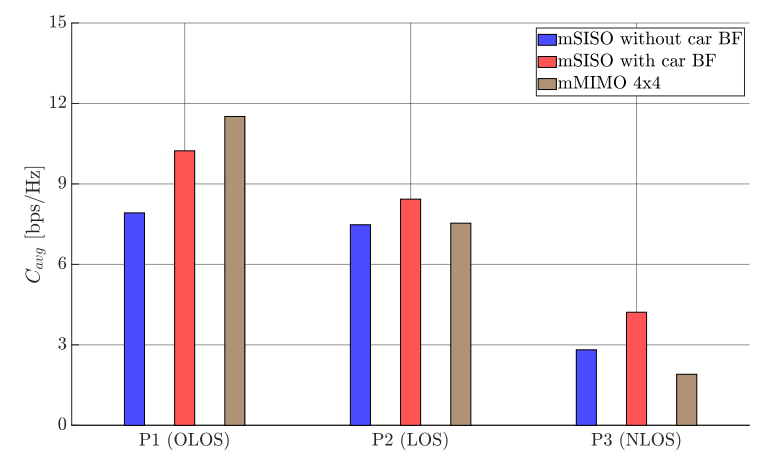

(a) $5.9 \mathrm{GHz}$

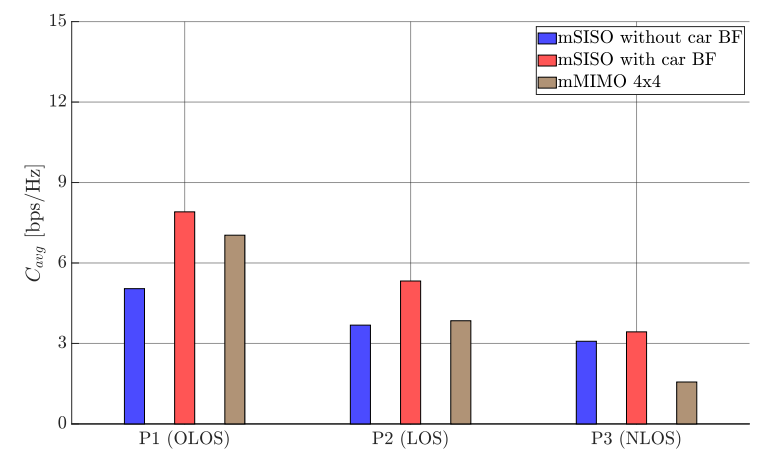

(b) $26 \mathrm{GHz}$.

Fig. 8. Average capacity within the focusing area at 5.9 and $26 \mathrm{GHz}$ for the three visibility regions.

\section{REFERENCES}

[1] T. Shimizu, V. Va et al., "Millimeter Wave V2X Communications: Use Cases and Design Considerations of Beam Management," in 2018 AsiaPacific Microwave Conference (APMC), Nov 2018, pp. 183-185.

[2] J. B. Kenney, "Dedicated Short-Range Communications (DSRC) Standards in the United States," Proceedings of the IEEE, vol. 99, no. 7, pp. 1162-1182, July 2011.

[3] 3GPP RP-181480, "New Study on NR V2X," 3GPP, SID new, June 2018.

[4] 3GPP TR 37.885, "Study on evaluation methodology of new Vehicleto-Everything V2X use cases for LTE and NR," 3GPP, draft TR, may 2018.

[5] ETSI, "ERM, Short Range Radar equipment operating in the $77 \mathrm{GHz}$ to $81 \mathrm{GHz}$ band, Part 1," European Telecommunications Standards Institute, Standard, 062009.

[6] C. K. Anjinappa and I. Guvenc, "Millimeter-Wave V2X Channels: Propagation Statistics, Beamforming, and Blockage," arXiv e-prints, $\mathrm{p}$ arXiv:1808.01001, Aug 2018.

[7] S. Mumtaz, J. Rodriguez, and L. Dai, "Chapter 1 - Introduction to mmWave massive MIMO," in mmWave Massive MIMO, Shahid Mumtaz and Jonathan Rodriguez and Linglong Dai, Ed. Academic Press, 2017, pp. $1-18$

[8] F. Challita, M. Martinez-Ingles et al., "Line-of-Sight Massive MIMO Channel Characteristics in an Indoor Scenario at $94 \mathrm{GHz}$," IEEE Access, vol. 6, pp. 62 361-62 370, 2018.

[9] E. Egea-Lopez, F. Losilla et al., "Vehicular Networks Simulation with Realistic Physics," IEEE Access, pp. 1-1, 2019.

[10] G. J. Foschini and M. J. Gans, "On limits of wireless communications in a fading environment when using multiple antennas," Wireless Personal Commun., vol. 6, no. 3, pp. 311-335, 1998.

[11] K. Haneda, J. Zhang et al., "5G 3GPP-Like Channel Models for Outdoor Urban Microcellular and Macrocellular Environments," in 2016 IEEE 83rd Vehicular Technology Conference (VTC Spring), May 2016, pp. $1-7$. 